\title{
Kinetic model for dose equivalent - an efficient way to predict systems response of irradiated cells
}

\author{
Stephan Scheidegger ${ }^{1}$, Rudolf Marcel Füchslin ${ }^{1,2}$ \\ scst@zhaw.ch \\ ${ }^{1}$ Centre of Applied Mathematics and Physics, Zurich University of Applied Sciences \\ Technikumstrasse 9, 8401 Winterthur \\ ${ }^{2}$ European Centre for Living Technology \\ S. Marco 2940, 30124 Venice, Italy
}

\begin{abstract}
The response of tumours onto ionizing radiation cannot be fully understood by commonly used radiobiological models. The reason may lie in the complex structure of the cellular systems which show a high degree of compartmentalisation and which is characterised by a network of interacting processes at different time scales. To access the dynamic response of cells onto radiation, compartmental models based on a biological dose equivalent can be used. Two different models ( $\Gamma$-LQ- and $\Gamma$-IRmodel) are used to fit experimental data of the clonogenic survival of irradiated cells at very high dose rates. The models reveal the correct dose rate dependence over a wide range of the parameter space when adapting the kinetic constants to the dose rate. This adaption could be an indication for the multi-scale structure of the system.
\end{abstract}

\section{Introduction}

Modern computers and modelling tools allow an efficient implementation and subsequent numerical treatment of biomedical models. Such simulations may be used for the exploration of the dynamic response of tumours onto anticancer treatment. This could lead to a quantitative basis for optimizing therapies, especially for novel or combined modalities such as radiotherapy and hyperthermia. However, such an approach requires to find a mathematical model or formulation describing the biological system in an appropriate way.

Biologically, the insufficiency of currently used models may be largely due to the complexity of the biological system: A whole variety of different intracellular mechanisms contribute to cellular repair and this at different time and dose scales. Not all of them are quantitatively (or even qualitatively) fully described.

In addition, some solid tumours exhibit enhanced radiosensitivity at very low doses $[1,2]$. Above a certain dose threshold, however, increased cellular repair can be observed for these cell lines which, on a first glance counter-intuitive, lowers radio 
sensitivity. This indicates a dose- and possibly dose rate dependent induction of repair mechanisms. The shortcomings of in vitro based models may also be caused by the inherent neglection of intercellular processes related to tissue dynamics, such as competition with host tissue, vascularisation and oxygenation, immune reactions and related inflammatory processes or the bystander effect [2].

Kinetic models at the level of cellular repair are described by many authors (e.g. Tobias [3] Curtis [4] and Carlone et al. [5] and Dale [6]). However, the extension to tissue interaction is difficult, since these models are based on the number of DNAlesions. To calculate the number of surviving cells, Poisson statistics is used. Since repopulation, competition or oxygen consumption depends on the population size, it would be favourable to use a corresponding formalism directly based on the numbers of tumour cells for models dedicated to the in vivo tumour dynamics. In addition, all these models do not reflect the fact that the cellular response cannot be explained by DNA lesions kinetics alone.

This article is addressing the following question: Is it possible to map the complex dynamic response of irradiated tumour cells onto simple kinetic models using a biological dose equivalent. Two kinetic approaches for modelling the dynamic tumour response onto radiation are presented. The models are based on a biological dose equivalent, which allows a flexible implementation of different aspects of dose rate dependent repair modifications and the interaction of intra- and extracellular processes. The models are compared to experimental data. The results indicate a high potential of kinetic models for investigating clinical aspects of anticancer therapy.

\section{Materials and Methods}

The basic idea behind the two presented models is to describe the reaction of tumor cells on radiation as a dynamic process taking account repair processes that take place in different time scales and interact in a non-trivial way. The models combine, necessarily in a heavily simplified manner, temporal changes of the tumour population (number of tumour cells $N=N(t)$ ), which may be composed of different subpopulations $i$ (number of tumour cells $N_{i}=N_{i}(t)$ ) and a kinetic model for a biological dose equivalent $\Gamma$. The unit of $\Gamma$ is that of the absorbed dose $D$ (energy per mass, $\mathrm{J} / \mathrm{kg}=\mathrm{Gy}$ ). Initially, the concept of a biological dose equivalent was proposed by Oliver [7]. Here, the dose equivalent $\Gamma$ is assumed to be proportional to the average number of unrepaired sublethal entities per a single cell produced by radiation. During irradiation, the energy deposition in tissue is much faster than the repair processes or the development of cellular damage. In this sense, $\Gamma=\Gamma(t)$ can be 
interpreted as transient dose, increasing with the dose rate $R=\dot{D}=d D / d t$ and decaying with the repair of the sublethal lesions.

In principle, the kinetic model for $\Gamma$ summarizes the biophysical and biochemical processes of energy deposition of radiation (ionisation, excitation), production of radicals and peroxides (in case of X-rays and / or electrons), reaction with biomolecules (DNA), lesions kinetics and repair of sublethal lesions. The equations describing the system (tumour cell population and kinetic dose equivalent model) are the following:

$$
\begin{aligned}
& \frac{d N_{i}}{d t}=f\left(N_{i}, N_{k}, \ldots, \Gamma\right) \\
& \frac{d N_{k}}{d t}=g\left(N_{i}, N_{k}, \ldots, \Gamma\right) \\
& \frac{d \Gamma}{d t}=R-h(\Gamma)
\end{aligned}
$$

Here, $h(\Gamma)$ is a function of $\Gamma$ representing the kinetics of repair of sublethal lesions, given at dose rate $R$. In the case of first order repair kinetics (as proposed by Oliver [7]), the repair rate is linear-proportional to the number of sublethal entities. Since an increasing number of experiments indicate a change of the half-time of repair, biexponential or binary repair (including two components of mono-exponential repair) is taken into account by several authors [8]. The observation of a fast repair shortly after irradiation and a following slower repair rate compared to exponential repair may also be explained by a second order process [6]. This implies, that the $\Gamma$-dependent function $h(\Gamma)$ in Eq.(1) is given by $h(\Gamma)=\gamma \cdot \Gamma_{1}^{2}$. For the following models, second order kinetic is used.

The quantity $\Gamma$ is related to the dose $D$ and the total dose $D_{\text {tot }}$ (= cumulative applied dose):

$$
\lim _{t \rightarrow \infty}\left[\int_{-\infty}^{t} h(\Gamma(\tau)) \cdot d \tau\right]=\lim _{t \rightarrow \infty}[D(t)]=D_{t o t}
$$




\subsection{The $\Gamma$ - LQ- model formulation}

In the $\Gamma$ - LQ- model formulation [9], only one tumour population (with the number of tumour cells $N=N(t)$ ) is used. Cell killing is described by two radiosensitivity coeffients $\alpha$ and $\beta$. The system (Eq.1) becomes:

$$
\begin{aligned}
& \frac{d N}{d t}=-(\alpha+2 \beta \Gamma) \cdot R \cdot N \\
& \frac{d \Gamma}{d t}=R-\gamma \Gamma^{2}
\end{aligned}
$$

At the beginning of an irradiation, $\Gamma$ is equal to the initial value $\Gamma(0)$. Integration of Eq. (3) using the constant value $\Gamma(0)$ leads to the surviving fraction: $S=e^{-\left(\alpha+2 \beta \Gamma_{1}(0)\right) \cdot D}$. The initial slope in the logarithmic diagram $(\log S=\ln S / \ln (10))$ for $\Gamma(0)=0 \mathrm{~Gy}$ for both first order and second order kinetics is $-\alpha / \ln (10)=-\alpha^{\prime}$. This is in agreement with the commonly used linear-quadratic (LQ) model.

In the case of $\gamma=0$, the quantity $\Gamma$ accumulates without outflow. Therefore $\Gamma$ is equal to the accumulated dose: $\Gamma(t)=D(t)$. In this case, integration of Eq. (5) leads to the classical LQ formalism with $N(D)=N_{0} \cdot e^{-\left(\alpha D+\beta D^{2}\right)}$.

For a single fraction with a constant dose rate $R, \Gamma(t)$ tends to an equilibrium (which will be reached for $t \rightarrow \infty$ ). For second order, the equilibrium level is $\Gamma_{e q}=\sqrt{R / \gamma}$. For this equilibrium state, Eq. (3) can be solved by separation and integration, since $\Gamma$ becomes constant $\Gamma(t) \rightarrow \Gamma_{e q}: N(D)=N_{0} \cdot e^{-\left(\alpha+2 \beta \Gamma_{e q}\right) \cdot D}$. The logarithmic of surviving fraction $S=N / N_{0}$ is given by $\ln S=-\left(\alpha+2 \beta \Gamma_{\text {eq }}\right) \cdot D$. The final slope in a logarithmic plot of the surviving fraction becomes constant:

$$
\left[\frac{d \ln S}{d D}\right]_{\Gamma \rightarrow \Gamma_{e q}}=-\left(\alpha+2 \beta \Gamma_{e q}\right)
$$

\subsection{A kinetic model for induced repair: The $\Gamma$ - IR- model}

The model formulation ( $\Gamma$ - IR- model) is based on two differential equations describing the radiation induced reduction of normal tumour cells $N_{1}$ and the recovery 
of lethally damaged cells $N_{2}$ (with the dose rate $R$ and a coefficient for radiosensitivity $\alpha$ ):

$$
\begin{aligned}
& \frac{d N_{1}}{d t}=-\alpha R N_{1}+\Theta\left(\Gamma, N_{2}\right) \\
& \frac{d N_{2}}{d t}=\alpha R \cdot\left(N_{1}-N_{2}\right)-\Theta\left(\Gamma, N_{2}\right)
\end{aligned}
$$

The term $\Theta\left(\Gamma, N_{2}\right)$ describes an additional, dose dependent repair mechanism with a characteristic dose equivalent $\Gamma_{c}$ :

$$
\Theta\left(\Gamma, N_{2}\right)=\vartheta \cdot e^{-\kappa\left(\Gamma-\Gamma_{c}\right)^{2}} \cdot N_{2}
$$

This approach can be justified by an activation of the additional repair process, which occurs for each cell at a different threshold dose [10]. It is assumed, that these thresholds can be characterized by a probability distribution with the maximum value $\Gamma_{c}$. The parameters $\alpha$ and $\Gamma_{c}$ can be determined directly from the logarithmic diagram $(\log S)$ of the surviving fraction $S=N_{1} / N_{1}(0), \alpha$ by the initial slope:

$$
\left[\frac{d \log S}{d D}\right]_{D \rightarrow 0}=-\alpha
$$

The parameter $\Gamma_{c}$ can be estimated from the local maximum (shoulder) of $\log S$, if low dose hypersensitivity appears. The parameters $\vartheta$ and $\kappa$ can be used for fitting experimental data. The final slope of the $\log S$ - curve is determined by the equilibrium of the dose equivalent $\Gamma_{e q}=\sqrt{R / \gamma}$. For this equilibrium condition, the following system of first order ODL results $d / d t\left[N_{i}\right]=m_{i k} N_{k}$ with the matrix $m_{i k}$ :

$$
m_{i k}=\left(\begin{array}{cc}
-\alpha R & \vartheta e^{-\kappa\left(\Gamma_{e q}-\Gamma_{C}\right)^{2}} \\
\alpha R & -\left(\alpha R+\vartheta e^{-\kappa\left(\Gamma_{e q}-\Gamma_{C}\right)^{2}}\right.
\end{array}\right)
$$

The final slope of the $\log S$ - curve is constant and dose rate dependent since the eigenvalues of the system Eq. (5) are:

$$
\lambda_{1,2}=-\alpha R-\frac{\vartheta}{2} \cdot e^{-\kappa\left(\sqrt{R / \gamma}-\Gamma_{C}\right)^{2}} \pm \frac{1}{2} \sqrt{4 \alpha R \cdot \vartheta e^{-\kappa\left(\sqrt{R / \gamma}-\Gamma_{C}\right)^{2}}+\vartheta^{2} e^{-2 \kappa\left(\sqrt{R / \gamma}-\Gamma_{C}\right)^{2}}}
$$




\section{Results}

Both models can be implemented easily by using graphical model editors such as Berkeley Madonna or equivalent. The results of the simulation using Berkeley Madonna (Berkeley Madonna Inc.) were compared to experimental data. The comparison of the $\Gamma$ - LQ- model with the in vitro data from Wells and Bedford [11] reveals a good fit to the logarithm of surviving fraction of irradiated C3H10T1/2 cells (Fig.1) over a wide range of the dose rate, if the kinetic constant $\gamma$ is adapted to the dose rate: $\gamma=a / R^{2}+b R+c$ with $a=1100 \mathrm{~Gy}^{3} / \mathrm{d}^{3}, \quad b=1.4 \cdot 10^{-2} \mathrm{~Gy}^{-2}$ and $c=5 \mathrm{~Gy}^{-1} \mathrm{~d}^{-1}$. The term $a / R^{2}$ is only important at dose rates below $100 \mathrm{~Gy} / \mathrm{d}(=0.07$ $\mathrm{Gy} / \mathrm{min})$. For dose rates above $1500 \mathrm{~Gy} / \mathrm{d}(=1.04 \mathrm{~Gy} / \mathrm{min})$, the curves are lying between the curve $d$ in Fig.1 and the curve for the LQ- model. In this area, a good fit to the model of Curtis is also reached with $\gamma=b R+c$.

The $\Gamma$ - LQ- model was used to fit data from irradiated glioblastoma cells at very high dose rate (Fig.2). The parameters were adjusted to the data set produced at the highest dose rate. Therefore, the model using a constant $\gamma$ fits the data not very adequately at intermediate dose rate $(R=288 \mathrm{~Gy} / \mathrm{d})$ but reveals a good fit for the very high dose rates $(R=8640 \mathrm{~Gy} / \mathrm{d}$ and $34560 \mathrm{~Gy} / \mathrm{d})$ with a constant value for $\gamma$.

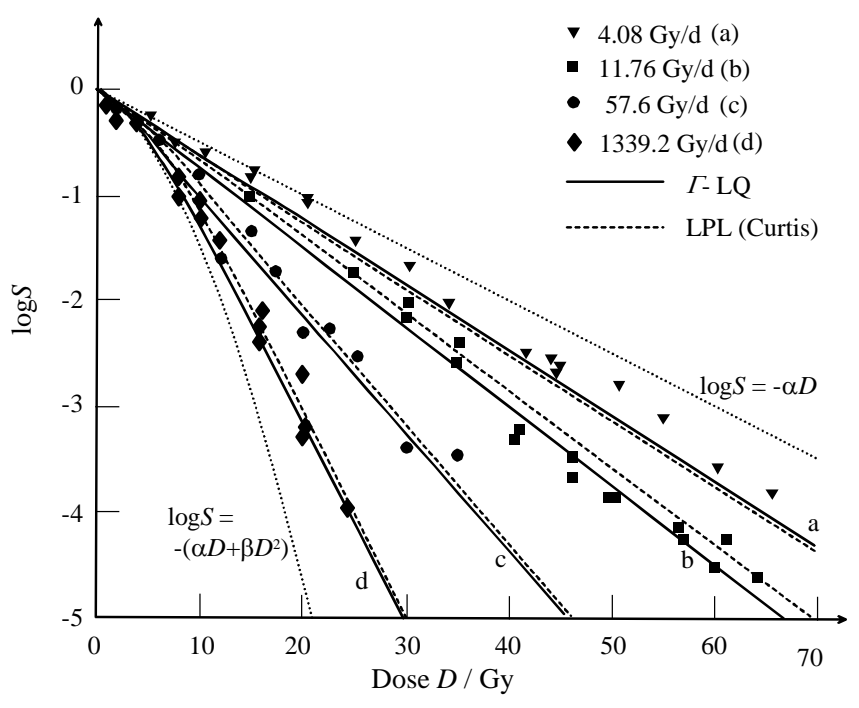

Figure 1: Comparison of the $\Gamma$-LQ formulation (second order kinetics, solid lines) with experimental data (C3H10T1/2 cells with $\alpha=0.1366 \mathrm{~Gy}^{-1}$ and $\beta=0.02 \mathrm{~Gy}^{-2}$, irradiated at different dose rates) from Wells and Bedford [11] and the model of Curtis (dashed lines): The dotted lines are at the right side the limit at low dose rate $(\log S=-\alpha D)$ and at the left side the common LQ-model. 


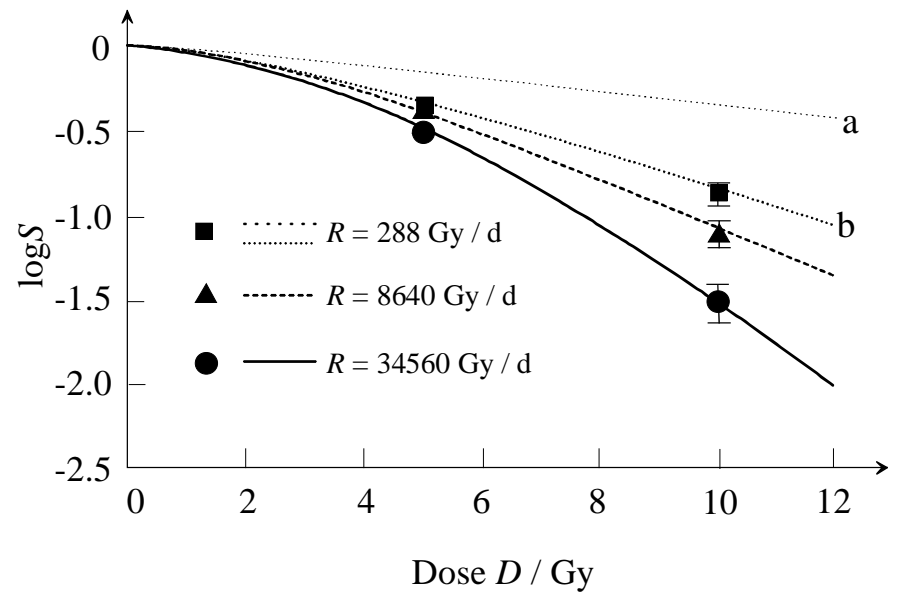

Figure 2: Surviving fraction of T98G- glioblastoma cells at different dose rates: For the high dose rate $(8.64 \mathrm{kGy} / \mathrm{d}$ and $34.56 \mathrm{kGy} / \mathrm{d})$, the $\Gamma$ - LQ- model can fit the experimental data with $\alpha=0.03 \mathrm{~Gy}^{-1}, \beta=0.04 \mathrm{~Gy}^{-2}$ and $\gamma=600 \mathrm{~d}^{-1}$; for the intermediate dose rate $(R=288 \mathrm{~Gy} / \mathrm{d})$, a good fit can only achieved by adapting the kinetic constant to $\gamma=45 \mathrm{~d}^{-1}$ (curve b, curve a with $\gamma=600 \mathrm{~d}^{-1}$ ).

The kinetic model for induced repair ( $\Gamma$ - IR- model, section 2.2 ) is able to reproduce the surviving fraction in the case of low dose hypersensitivity as observed e.g. for irradiated breast cancer cells (Fig.3). Also the distinct behaviour of clonogenic survival of apoptotic versus non-apoptotic cell death can be explained by the $\Gamma$ - IRmodel. The $\Gamma$-IR-model seems to fit a broad spectrum of radiobiological observations.

To investigate the dose rate dependence, the $\Gamma$ - IR- model was used to fit the in vitro data of the T98G glioblastoma cells shown in Fig.2 [12]. In Fig.4, a good fit of the experimental data can be achieved by adapting both, $\vartheta$ and $\gamma$ to the dose rate. The value of parameter $\vartheta$ is different for all three dose rates $\left(\vartheta=10^{3} \mathrm{~d}^{-1}\right.$ for $R=288$ $\mathrm{Gy} / \mathrm{d} ; \vartheta=3 \cdot 10^{4} \mathrm{~d}^{-1}$ for $R=8.64 \mathrm{kGy} / \mathrm{d}$ and $\vartheta=1.6 \cdot 10^{5} \mathrm{~d}^{-1}$ for $\left.R=34.56 \mathrm{kGy} / \mathrm{d}\right)$, whereas the kinetic constant $\gamma$ has the same value $\left(\gamma=540 \mathrm{~Gy}^{-1} \mathrm{~d}^{-1}\right)$ for $R=8.64$ $\mathrm{kGy} / \mathrm{d}$ and $R=34.56 \mathrm{kGy} / \mathrm{d}$. Only for $R=288 \mathrm{~Gy} / \mathrm{d}$, the value is set to $\gamma=23 \mathrm{~Gy}^{-1} \mathrm{~d}^{-1}$. 


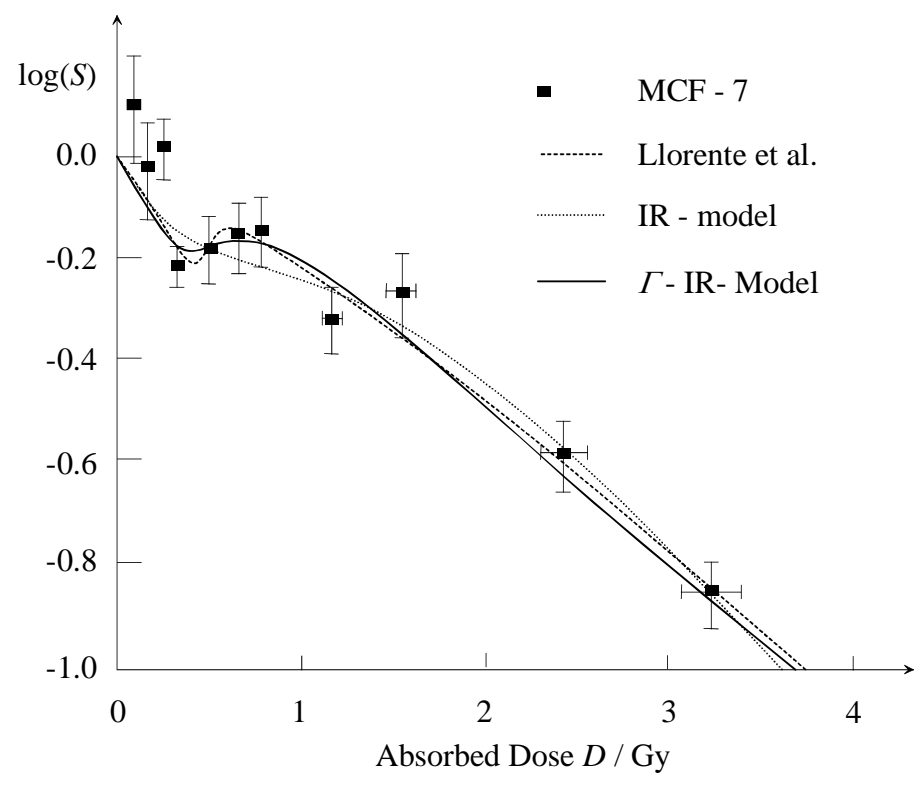

Figure 3: Comparison of the proposed $\Gamma$ - IR- Model with the model of Guirado Lllorente et al. [10], the IR-Model and experimental data (irradiated multi cellular spheroids of breast cancer MCF-7 cell line [10]); Parameters: $\alpha=2.5 \mathrm{~Gy}^{-1}$, $\Gamma_{C}=0.6 \mathrm{~Gy}, \kappa=14 \mathrm{~Gy}^{-2}, \gamma=0.729 \mathrm{~Gy}^{-1} \mathrm{~min}^{-1}$ and $\vartheta=20.833 \mathrm{~min}^{-1}$.

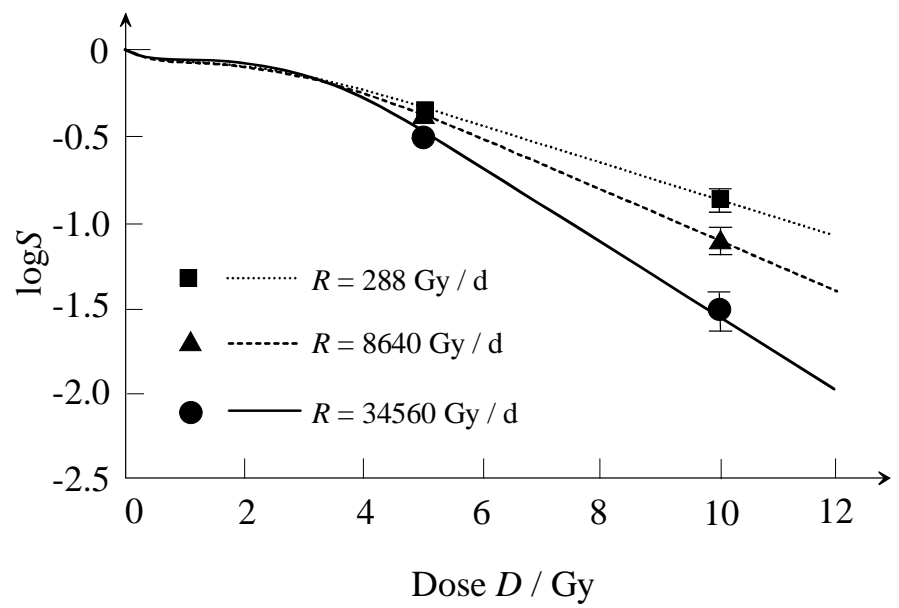

Figure 4: Comparison of the proposed $\Gamma$ - IR- Model with experimental data (T98G glioblastoma [12]); Parameters: $\alpha=0.5 \mathrm{~Gy}^{-1}, \Gamma_{C}=1.2 \mathrm{~Gy}, \kappa=0.45 \mathrm{~Gy}^{-2}, \vartheta$ and $\gamma$ are adapted to the dose rate. 


\section{Discussion and Conclusions}

The use of a dose equivalent concept leads to a flexible structure of model formulation. Both models are able to fit the observed dose rate dependence of experimental data although more experimental data for fitting should be available (especially Fig.2 and 4). But in both models, the kinetic constant has to be adapted to the dose rate to achieve a good fit over the whole range of dose rate. Only for a dose rate above 8.64 $\mathrm{kGy} / \mathrm{d}=6 \mathrm{~Gy} / \mathrm{min}$, the $\Gamma$ - LQ- model reveals a good fit with three dose rate independent constants. The adaption of the constants to the dose rate in addition to the dose rate dependency given by the kinetic model for dose equivalent indicates that different mechanism at different time scales contribute to the repair and cell death processes. The use of a dose equivalent to describe cellular damages is a simplistic approximation of a very complex system, but it allows approaching the dynamic system behaviour by a kinetic model structure.

In addition to the intracellular processes, the communication between cells are important for the tumour (tissue) response (e.g., bystander effect [1,2]). Damaged cell do not only repair themselves, but somehow (possibly via cytokines) transmit the information about the fact that they are damaged to other cells, probably in their direct proximity. This "cross-talk" on the tissue-level is the base for the appearance of emergent behaviour, as it is well known from many physical systems, e.g. near phase transitions.

This seems to be similar to thermodynamics, where a very diverse and complicated microscopic dynamics nevertheless leads to a macroscopic phenomenology which is well described in terms such as internal energy, temperature etc. However, comparing cellular dynamics with thermodynamics is only sensible on a higher level of abstraction. Cellular processes are generically not in equilibrium, whereas the concepts of thermodynamics only apply to equilibrium situations (in some cases stationary processes, which are globally not in equilibrium but allow sensibly speaking of local equilibria, are also well described by thermodynamic quantities). But there are structural similarities too: Thermodynamic systems are most often described by surprisingly few parameters and this is also a tacit (and experimentally justified) assumption of cellular modelling, probably caused by the fact that cellular as well as thermodynamic systems (e.g. phases) exhibit robust emergent behaviour. Today, the observables of cellular systems are mostly chosen by criteria of experimental accessibility. In future, further effort should be spend to identify system parameters that represent the true dynamics of cellular systems as precise as possible. There may be a price to pay, because according measurement may be difficult. The benefit, 
however, reliable prediction of dynamics using comparably simple models, may bear a considerable potential for anticancer treatment.

\section{References}

[1] Lambin, P., Malaise, E.P., Joiner, M.C.: Might intrinsic radioresistance of tumour cells be induced by radiation? Int. J. Radiat. Oncol. Biol. 69(1996), 279-90.

[2] Mothersill, C., Seymor, C.B., Joiner, M.C.: Relationship between radiation - induced low dose hypersensitivity and the bystander effect. Radiat. Res. 157(2002), 526-32.

[3] Tobias, C.A.: The repair-misrepair model in radiobiology: Comparison to other methods. Radiat. Res. 104(1985), S77-95.

[4] Curtis, S.B.: Lethal and potentially lethal lesions induced by radiation - A Unified Repair Model. Radiat. Res. 106(1986), 252-70.

[5] Carlone, M.C., Wilkins, D., Raaphorst, G.P.: The modified linear-quadratic model of Guerrero and Li can be derived from amechanistic basis and exhibits linearquadratic-linear behaviour. Phys. Med. Biol. 50(2005), L9-13

[6] Dale, R.G., Fowler, J.F., Jones, B.: A new incomplete-repair model based on a reciprocal-time pattern of sublethal damage repair. Acta Oncol. 38 (1999), 919-29.

[7] Oliver, R.A.: comparison of the effects of acute and protracted gamma-radiation on the growth of seedlings of Vicia faba. Int. J. Radiat. Biol. 8(1964), 475-88.

[8] Fowler, J.F.: Repair between dose fractions: A simpler method of analyzing and reporting apparently biexponential repair. Radiat. Res. 158(2002), 141-51.

[9] Scheidegger, S., Lutters, G, Bodis, S.: A LQ-based kinetic model formulation for exploring dynamics of treatment response of tumours in patients. Z. Med. Phys. (2011), in print: DOI: 10.1016/j.zemedi.2010.11.001

[10] Guirado Llorente, D., Aranda, M., Ortiz Seidel, M., Mesa Pérez, J.A., Vega Fernandez J.M.D.L., Martinez Luna, R.J., Zamora Ardoy, L.I., Villalobos Torres, M., Lallena, A.M.: Low dose hypersensitivity in mulicellular tumour spheroids. Radiother. Oncol. 96 (2010), Supl. 1, 607-8.

[11] Wells, R.L., Bedford, J.S.: Dose rate effects in mammalian cells.IV. Repairable and nonrepairable damage in noncyclic C3H10T1/2 cells. Radiat. Res. 94(1983), 105-34

[12] Zaugg, K., Lohse, I., Lang, S., Hrbacek, I., Sanchez Macedo, N., Feng, J., Lütolf U.M.: High dose radiation using a flattening filter free beam reduces cancer cell survival in vitro. Proc. of $15^{\text {th }}$ scientific annual meeting of SASRO (2011), 69 